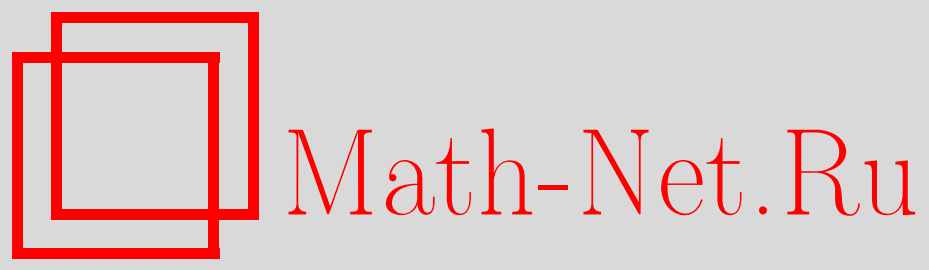

В. П. Маслов, О быстром изменении магнитного поля для заряженного совершенного газа при больших числах Рейнольдса, Функи. анализ и его прил., 1996, том 30, выпуск 3, 47-61

DOI: https://doi.org/10.4213/faa537

Использование Общероссийского математического портала MathNet.Ru подразумевает, что вы прочитали и согласны с пользовательским соглашением

http://www . mathnet.ru/rus/agreement

Параметры загрузки:

IP : 44.207 .124 .84

26 апреля 2023 г., 02:52:40

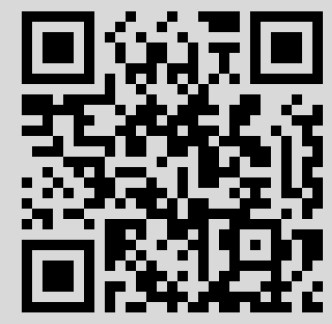


Функииональный анализ и его приложения

1996, т. 30, вып. 3, с. 47-61

УДК 517.9

\title{
О быстром изменении магнитного поля для заряженного совершенного газа при больших числах Рейнольдса
}

\author{
(C) $1996 . \quad$ В. П. МАСлов
}

Проблема детерминистического объяснения эффекта роста магнитного поля (проблема «быстрого магнитного динамо»), как правило, математиками формулируется лишь для линейных уравнений Максвелла с большим магнитным числом Рейнольдса $\operatorname{Re}_{M}$, т. е. скорость ионизированного газа считается заданной и рассматривается рост поля при $t \rightarrow \infty$. Эта проблема так и не была решена. В физической реальности $\operatorname{Re}_{M} \sim \operatorname{Re}$, где $\operatorname{Re}$ - гидродинамическое число Рейнольдса. Поэтому необходимо считать, что оба числа Рейнольдса велики и скорость газа существенно зависит от поля. Мы рассмотрим здесь ситуацию сильной анизотропии, т.е. ситуацию, когда масштаб в направлении некоторого векторного поля много меньше в начальный момент, чем масштаб в перпендикулярной $\mathrm{k}$ нему плоскости. Соответственно будем обозначать через $H_{\perp}, v_{\perp}$ проекции напряженности магнитного поля и скорости на эту плоскость, а через $H_{\|}, v_{\|}$их проекции вдоль этого векторного поля в ситуации, когда в начальный момент $H_{\|}^{0} \sim 1 / \sqrt{\operatorname{Re}}$. Оказывается, что для совершенного газа при $H_{\perp}^{0}=0$ в начальный момент магнитное поле уже за малое время $t$ вырастает при $\operatorname{Re} \rightarrow \infty$ до величины порядка $O\left(t^{2}\right) \gg 1 / \sqrt{\mathrm{Re}}$ (эффект динамо), и, более того, изменение начального значения $H_{\|}^{0}$ на величину порядка $1 / \sqrt{\mathrm{Re}}$ дает изменение порядка $O(t)$ средней скорости $v_{\perp}$, т. е. для $v_{\perp}$ имеет место рост, еще более быстрый, чем у магнитного поля (новый эффект).

Рассмотрим уравнения магнитной газодинамики с учетом эффектов переноса тепла:

$$
\begin{gathered}
\rho\left(\frac{\partial v^{i}}{\partial t}+\langle v, \nabla\rangle v^{i}\right)=-\frac{\partial}{\partial x_{i}} P-\frac{1}{4 \pi}(H \times \operatorname{rot} H)^{i} \\
+\frac{1}{\operatorname{Re}} \frac{\partial}{\partial x_{k}}\left\{\mu\left(\frac{\partial v^{i}}{\partial x_{k}}+\frac{\partial v^{k}}{\partial x_{i}}-\frac{2}{3} \delta_{i k} \operatorname{div} v\right)\right\}+\frac{1}{\operatorname{Re}} \frac{\partial}{\partial x_{i}}(\zeta \operatorname{div} v)+F_{N} \\
\frac{\partial \rho}{\partial t}+\operatorname{div}(\rho v)=0 \\
\frac{\partial H}{\partial t}=\operatorname{rot}(v \times H)-\frac{1}{\operatorname{Re}_{M}} \operatorname{rot}(\nu \operatorname{rot} H), \\
\operatorname{div} H=0
\end{gathered}
$$




$$
\begin{gathered}
\rho\left(\frac{\partial T}{\partial t}+\langle v, \nabla T\rangle\right)=(1-\gamma) \rho T \operatorname{div} v+\frac{1}{\operatorname{Re}} \operatorname{div}(\varkappa \nabla T)+\frac{1}{\operatorname{Re}_{M}} \frac{\nu}{4 \pi}(\operatorname{rot} H)^{2} \\
+\frac{1}{\operatorname{Re}} \frac{\mu}{2} \frac{\partial v^{i}}{\partial x_{k}}\left(\frac{\partial v^{i}}{\partial x_{k}}+\frac{\partial v^{k}}{\partial x_{i}}-\frac{2}{3} \delta_{i k} \operatorname{div} v\right)+\frac{\zeta}{\operatorname{Re}}(\operatorname{div} v)^{2} \\
P=k \rho T .
\end{gathered}
$$

Здесь $v=\left(v^{1}, v^{2}, v^{3}\right)$ - вектор скорости, $\rho$ - плотность, $P$ - давление, $T-$ температура, $H$ - вектор напряженности магнитного поля, $\mu=\mu(\rho, T)>0$, $\zeta=\zeta(\rho, T)>0, \varkappa=\varkappa(\rho, T)>0$ - нормированные на число Рейнольдса Re коэффициенты вязкости и теплопроводности, $\nu=\nu(\rho, T)>0-$ нормированный на число Рейнольдса $\operatorname{Re}_{M}$ коэффициент электропроводности, $k>0, \gamma>0$ - физические константы, $\delta_{i k}$ - символ Кронекера, причем по всем повторяюшимся индексам предполагается суммирование. Угловыми скобками обозначено скалярное произведение в $\mathbb{R}^{3}$. Уравнения рассматриваются в неограниченной области.

Функцию $F_{N}=F_{N}\left(x, t, \mathrm{Re}^{-1}\right)$ будем называть малыми (флуктуационными) массовыми силами класса $N$, если для целого положительного $N$ и любого $K$ выполнено неравенство

$$
\max _{0 \leqslant t \leqslant t_{0}}\left|F\left(x, t, \operatorname{Re}^{-1}\right)\right| \leqslant \operatorname{Re}^{-N} \frac{C_{N}}{\left(1+|x|^{2}\right)^{K}},
$$

где $C_{N}=$ const, $t_{0}=$ const .

Уравнения (1)-(6) рассматриваются при больших гидродинамических и магнитных числах Рейнольдса. Будем считать, что эти числа удовлетворяют соотношению $\operatorname{Re}_{M} / \operatorname{Re} \leqslant c \operatorname{Re}_{M}^{\beta}$, где $c-$ некоторая константа, а $0 \leqslant \beta<1 / 2$. Введем малый параметр $\varepsilon=\operatorname{Re}_{M}^{\beta-(1 / 2)}$. Все коэффициенты $\mu, \zeta, \nu$ и $\varkappa$ будем считать гладкими функциями плотности и температуры.

Пусть начальные данные для системы (1)-(5) имеют вид

$$
\begin{gathered}
\left.v\right|_{t=0}=v^{0}\left(\frac{\Phi^{0}(x, \varepsilon)}{\varepsilon}, x, \varepsilon\right),\left.\quad \rho\right|_{t=0}=\rho^{0}\left(\frac{\Phi^{0}(x, \varepsilon)}{\varepsilon}, x, \varepsilon\right), \\
\left.T\right|_{t=0}=T^{0}\left(\frac{\Phi^{0}(x, \varepsilon)}{\varepsilon}, x, \varepsilon\right), \\
\left.H\right|_{t=0}=\varepsilon H^{0}\left(\frac{\Phi^{0}(x, \varepsilon)}{\varepsilon}, x, \varepsilon\right),
\end{gathered}
$$

где $\Phi^{0}(x, \varepsilon)$ - бесконечно дифференцируемая по $x$ функция, являющаяся многочленом по $\varepsilon$, причем $\left|\nabla \Phi^{0}(x, 0)\right| \geqslant c>0$, где $c$ - некоторая константа. Функщии $v^{0}(\eta, x, \varepsilon), H^{0}(\eta, x, \varepsilon), \rho^{0}(\eta, x, \varepsilon)$ и $T^{0}(\eta, x, \varepsilon)$ бесконечно дифференцируемы по всем своим аргументам, $2 \pi$-периодичны по $\eta$ и являются многочленами степени $m$ по $\varepsilon$. Число $m$ может быть сколь угодно велико. Начальные векторы скорости и напряженности магнитного поля $v^{0}$ и $H^{0}$ финитны по $x$, а начальные плотность и температура $\rho^{0}$ и $T^{0}$ строго положительны и вне некоторой ограниченной области равны константам. 
Представим начальные данные в виде

$$
\begin{gathered}
\Phi^{0}(x, \varepsilon)=\sum_{i=0}^{m} \Phi_{i}^{0}(x) \varepsilon^{i}, \quad v^{0}(\eta, x, \varepsilon)=\sum_{i=0}^{m} v_{i}^{0}(\eta, x) \varepsilon^{i}, \\
H^{0}(\eta, x, \varepsilon)=\sum_{i=0}^{m-1} H_{i+1}^{0}(\eta, x) \varepsilon^{i}
\end{gathered}
$$

Пусть функция $f(\eta, x, t, \varepsilon)$ является $2 \pi$-периодической по $\eta$; через $f_{\text {av }}$ будем обозначать ее среднее значение по периоду: $f_{\mathrm{av}}=(1 / 2 \pi) \int_{0}^{2 \pi} f(\eta, x, t, 0) d \eta$.

Очевидно, что $f_{\text {av }}(x)$ равно слабому пределу (т. е. осреднению) функции $f(\Phi(x, \varepsilon) / \varepsilon, x, \varepsilon)$ при $\varepsilon \rightarrow 0$. Будем писать $f(\Phi(x, \varepsilon) / \varepsilon, x, \varepsilon) \rightarrow_{\varepsilon \rightarrow 0} f_{\text {av }}(x)$, если $\lim _{\varepsilon \rightarrow 0} \int \varphi(x) f(\Phi(x, \varepsilon) / \varepsilon, x, \varepsilon) d x=\int \varphi(x) f_{\mathrm{av}}(x) d x$ для любой функции $\varphi(x) \in C_{0}^{\infty}$.

Начальную скорость будем представлять в виде суммы осредненной и осциллирующей частей: $v_{i}^{0}(\eta, x)=u_{i}^{0}(x)+a_{i}^{0}(\eta, x)$, где $\left(a_{i}^{0}\right)_{\mathrm{av}}=0$. Обозначим $\left\langle a_{i}^{0}, \nabla \Phi_{0}^{0}\right\rangle$ через $a_{i \|}^{0}$, a $\left\langle H_{i}^{0}, \nabla \Phi_{0}^{0}\right\rangle$ через $H_{i \|}^{0}$.

Предполагаем, что для начальных данных выполнены следующие соотношения:

$$
\begin{gathered}
\operatorname{div} H^{0}\left(\frac{\Phi^{0}(x, \varepsilon)}{\varepsilon}, x, \varepsilon\right)=0, \quad \frac{\partial}{\partial \eta} P^{0}(\eta, x, 0)=0, \\
a_{0 \|}^{0}=0, \quad a_{1 \|}^{0}=-\left\langle a_{0}^{0}, \nabla \Phi_{1}^{0}\right\rangle+\frac{H_{1 \|}^{0}\left\langle a_{0}^{0}, B^{0}\right\rangle}{4 \pi \gamma P^{0}+\left|B^{0}\right|^{2}}+F_{1}, \\
a_{i \|}^{0}=F_{i}, \quad i=2, \ldots, m,
\end{gathered}
$$

где $P^{0}=k \rho^{0} T^{0}$, а $F_{i}, i=1, \ldots, m$, 一 некоторые функции, зависящие от других начальных условий. Их вид см. в (35). Таким образом, давление в начальный момент не осциллирует. Условия (10) означают, что осциллирующая составляющая начальной скорости ортогональна быстрому изменению начальных данных.

Будем считать, что коэффищиенты вязкости и электропроводности $\mu, \zeta, \nu$ зависят от температуры линейно, а коэффициент теплопроводности $\varkappa$ от температуры не зависит.

ТЕОРемА 1. Для любых сколь угодно большого $N$ и сколь угодно малого $\delta>0$ имеются такие малье массовье сильи $F_{N}\left(t, x, \mathrm{Re}^{-1}\right)$ класса $N$, что существуют решения $v(x, t, \varepsilon), \rho(x, t, \varepsilon), H(x, t, \varepsilon), T(x, t, \varepsilon), P(x, t, \varepsilon)$ задачи (1)-(10) при $t \leqslant t_{0} \leqslant \mathrm{Re}^{-\delta}$, причем

$$
\operatorname{Re}^{2 \delta} H\left(x, t_{0}\right) \underset{\operatorname{Re} \rightarrow \infty}{\rightarrow} \frac{1}{2}\left(\operatorname{rot}\left(\left(H_{1 \|}^{0}\right)_{\mathrm{av}}\left(a_{0}^{0} \times \frac{\partial a_{0}^{0}}{\partial \eta}\right)\right){ }_{\mathrm{av}}\right)
$$

Рассмотрим более общие начальные данные, а именно вместо (8) возьмем

$$
\left.H\right|_{t=0}=B^{0}(x)+\varepsilon H^{0}\left(\frac{\Phi^{0}(x, \varepsilon)}{\varepsilon}, x, \varepsilon\right), \quad \text { причем }\left\langle B^{0}, \nabla \Phi^{0}\right\rangle=0 .
$$


Прибавим к начальным данным (7), (11) малую величину порядка $O(\varepsilon)$. А именно, пусть новые начальные данные имеют вид

$$
\begin{gathered}
\left.\check{H}\right|_{t=0}=\left.H\right|_{t=0}+\varepsilon \breve{H}_{1}^{0}=B^{0}+\varepsilon H^{0}+\varepsilon \breve{H}_{1}^{0}, \\
\left.v\right|_{t=0}=v^{0}+\varepsilon \frac{\check{H}_{1 \|}^{0}\left\langle a_{0}^{0}, B^{0}\right\rangle}{4 \pi \gamma P^{0}+\left|B^{0}\right|^{2}},\left.\quad \check{\rho}\right|_{t=0}=\rho^{0},\left.\quad \check{T}\right|_{t=0}=T^{0},
\end{gathered}
$$

где $\partial \breve{H}_{1}^{0} / \partial \eta=0$. Имеет место следующая теорема.

Теорема 2. Для сколь угодно большого $N$ и сколь угодно малого $\delta>0$ имеютсл такие малье массовые силь $F_{N}$ класса $N$, что существуют решения $v(x, t, \varepsilon), \rho(x, t, \varepsilon), H(x, t, \varepsilon), T(x, t, \varepsilon), P(x, t, \varepsilon)$ уравнений $(1)-(6)$ с начальными данными (7), (9)-(11) и решения $\check{v}(x, t, \varepsilon), \check{\rho}(x, t, \varepsilon), \check{H}(x, t, \varepsilon)$, $\breve{T}(x, t, \varepsilon), \check{P}(x, t, \varepsilon)$ уравнений (1)-(6) с начальными данньли (12), (9), (10), maкue, чmo

$$
\operatorname{Re}^{\delta}\left(\check{v}_{\mathrm{av}}\left(x, t_{0}\right)-v_{\mathrm{av}}\left(x, t_{0}\right)\right) \underset{\operatorname{Re} \rightarrow \infty}{\rightarrow} \frac{\left(a_{0}^{0}\left\langle a_{0}^{0}, B^{0}\right\rangle\right)_{\mathrm{av}} \check{H}_{1 \|}^{0}}{4 \pi \gamma P^{0}+\left|B^{0}\right|^{2}}
$$

В процессе доказательства теоремы такие решения будут предъявлены.

Мы докажем ниже более общее и более громоздкое утверждение, которое сформулируем в виде леммы.

ЛЕмма. Для сколь угодно большого $N$ и сколь угодно малого $\delta>0$ имеются такие малье массовые сильи $F_{N}$ класса $N$, что существуют решения $v(x, t, \varepsilon), \rho(x, t, \varepsilon), H(x, t, \varepsilon), T(x, t, \varepsilon), P(x, t, \varepsilon)$ задачи (1)-(7), (9)-(11). Для любой функиии $\varphi(x) \in C_{0}^{\infty}$ имеет место соотношение

$$
\begin{aligned}
\lim _{\mathrm{Re} \rightarrow \infty} \operatorname{Re}^{2 \delta} \int \varphi(x)\left(H\left(x, t_{0}\right)-\right. & \left.\widetilde{H}\left(x, t_{0}\right)\right) d x \\
& =\frac{1}{2} \int \varphi(x) \operatorname{rot}\left\{\left(H_{1 \|}^{0}\right)_{\mathrm{av}}\left(a_{0}^{0} \times \frac{\partial a_{0}^{0}}{\partial \eta}\right)_{\mathrm{av}}\right\} d x
\end{aligned}
$$

где $\widetilde{H}$ удовлетворяет при $t \leqslant t_{0}$ уравнениям Рейнольдса:

$$
\begin{gathered}
\tilde{\rho}\left(\frac{\partial \tilde{v}}{\partial t}+\langle\tilde{v}, \nabla\rangle \tilde{v}\right)+\left(\frac{\tilde{\rho}}{\rho^{0}}\right)_{\mathrm{av}}\left(\nabla \widetilde{P}+\frac{1}{4 \pi}(\widetilde{H} \times \operatorname{rot} \widetilde{H})\right)=G_{1}, \\
\frac{\partial \tilde{\rho}}{\partial t}+\operatorname{div}(\tilde{\rho} \tilde{v})=G_{2}, \quad \frac{\partial \widetilde{H}}{\partial t}-\operatorname{rot}(\tilde{v} \times \widetilde{H})=G_{3}, \quad \operatorname{div} \widetilde{H}=0, \\
\tilde{\rho}\left(\frac{\partial \widetilde{T}}{\partial t}+\langle\tilde{v}, \nabla \widetilde{T}\rangle+(\gamma-1) \widetilde{T} \operatorname{div} \tilde{v}\right)=G_{4}, \\
\widetilde{P}=k \tilde{\rho} \widetilde{T},\left.\quad \tilde{v}\right|_{t=0}=u_{0}^{0},\left.\quad \tilde{\rho}\right|_{t=0}=\left(\rho^{0}\right)_{\mathrm{av}}, \\
\left.\widetilde{H}\right|_{t=0}=B^{0},\left.\quad \widetilde{T}\right|_{t=0}=\left(T^{0}\right)_{\mathrm{av}},
\end{gathered}
$$


$2 \partial e^{1}$

$$
\begin{aligned}
G_{1}=- & \tilde{\rho}\left(\left\langle a_{0}^{0}, \nabla\right\rangle a_{0}^{0}\right)_{\mathrm{av}}-\tilde{\rho}\left(\gamma k \rho^{0} T^{0}+\frac{1}{4 \pi}\left|B^{0}\right|^{2}\right)^{-1} \\
& \times\left(a _ { 0 } ^ { 0 } \left(\frac{1}{4 \pi}\left(\left(H_{1 \|}^{0}\right)_{\mathrm{av}}\left\langle\frac{\partial a_{0}^{0}}{\partial \eta}, B^{0}\right\rangle+\left\langle\operatorname{rot}\left(a_{0}^{0} \times B^{0}\right), B^{0}\right\rangle\right)\right.\right. \\
& \quad+k \gamma\left(\rho^{0} T^{0} \operatorname{div} v^{0}+\left|\nabla \Phi^{0}\right|^{2}\left(\frac{\partial}{\partial \eta} \varkappa\left(\rho^{0}, T^{0}\right) \frac{\partial T^{0}}{\partial \eta}\right.\right. \\
& \left.\left.\left.\left.+\mu\left(\rho^{0}, T^{0}\right)\left|\frac{\partial a_{0}^{0}}{\partial \eta}\right|^{2}\right)\right)-k\left\langle v^{0}, \nabla \rho^{0} T^{0}\right\rangle\right)\right)_{\mathrm{av}} \\
+ & \frac{\nabla \Phi^{0}}{\left|\nabla \Phi^{0}\right|^{2}}\left(\left(\frac{1}{\rho^{0}}\right)_{\mathrm{av}}-\frac{1}{\rho_{\mathrm{av}}^{0}}\right)\left(\rho^{0} \Pi\left(2\left\langle\nabla \Phi^{0},\left\langle a_{0}^{0}, \nabla\right\rangle v_{\mathrm{av}}^{0}\right\rangle-\left\langle a_{0}^{0}, \Phi^{0^{\prime \prime}} a_{0}^{0}\right\rangle\right)\right)_{\mathrm{av}} \\
+ & \left|\nabla \Phi^{0}\right|^{2}\left(\frac{\mu\left(\rho^{0}, T^{0}\right)\left(\partial a_{0}^{0} / \partial \eta\right) \partial \rho^{0} / \partial \eta}{\left(\rho^{0}\right)^{2}}\right)_{\mathrm{av}}
\end{aligned}
$$

$G_{2}=-\operatorname{div}\left(a_{0}^{0} \rho^{0}\right)_{\mathrm{av}}$,

$$
\begin{aligned}
G_{3}=t & \left(\operatorname{rot}\left(a_{0}^{0} \times\left(\left\langle B^{0}, \nabla\right\rangle a_{0}^{0}-\left\langle a_{0}^{0}, \nabla\right\rangle B^{0}\right)\right)_{\mathrm{av}}\right. \\
& +\operatorname{rot}\left(a_{0}^{0} \times B^{0}\left(( \gamma k \rho ^ { 0 } T ^ { 0 } + \frac { | B ^ { 0 } | ^ { 2 } } { 4 \pi } ) ^ { - 1 } \left(\frac{1}{4 \pi}\left\langle\operatorname{rot}\left(a_{0}^{0} \times B^{0}\right), B^{0}\right\rangle\right.\right.\right. \\
& +k \gamma\left(\rho^{0} T^{0} \operatorname{div} a_{0}^{0}+\left|\nabla \Phi^{0}\right|^{2} \frac{\partial}{\partial \eta} \varkappa\left(\rho^{0}\right) \frac{\partial T^{0}}{\partial \eta}+\mu\left(\rho^{0}, T^{0}\right)\left|\frac{\partial a_{0}^{0}}{\partial \eta}\right|^{2}\right) \\
& \left.\left.\left.\left.-k\left\langle a_{0}^{0}, \nabla \rho^{0} T^{0}\right\rangle\right)+\operatorname{div} a_{0}^{0}\right)\right)\right),
\end{aligned}
$$

$$
G_{4}=\gamma\left|\nabla \Phi_{0}^{0}\right|^{2}\left(\mu\left(\rho^{0}, T^{0}\right)\left|\frac{\partial a_{0}^{0}}{\partial \eta}\right|^{2}\right)+T_{\mathrm{av}}^{0} \operatorname{div}\left(a_{0}^{0}\left(\rho^{0}-\rho_{\mathrm{av}}^{0}\right)\right)_{\mathrm{av}},
$$

причем $\Pi f=f-f_{\mathrm{av}}$, a $\Phi^{0^{\prime \prime}}$ - матрииа Гесса функции $\Phi^{0}$.

ДокАЗАТЕЛЬСтво. На первом этапе доказательства леммы построим формальное асимптотическое по $\varepsilon$ решение уравнений (1)-(6) с начальными данными (7), (11). Такое решение мы ищем в виде

$$
\begin{aligned}
v_{n}(\eta, x, t, \varepsilon)=\sum_{i=0}^{n} \mathscr{V}_{i}(\eta, x, t) \varepsilon^{i}, & \rho_{n}(\eta, x, t, \varepsilon)=\sum_{i=0}^{n} \mathscr{R}_{i}(\eta, x, t) \varepsilon^{i}, \\
H_{n}(\eta, x, t, \varepsilon)=\sum_{i=0}^{n} \mathscr{H}_{i}(\eta, x, t) \varepsilon^{i}, & P_{n}(\eta, x, t, \varepsilon)=\sum_{i=0}^{n} \mathscr{P}_{i}(\eta, x, t) \varepsilon^{i},
\end{aligned}
$$

где $\eta=\Phi_{0}(x, t) / \varepsilon+\Phi_{1}(x, t)+\cdots+\varepsilon^{n} \Phi_{n}(x, t),\left.\eta\right|_{t=0}=\Phi^{0}(x, t) / \varepsilon$, функции

${ }^{1}$ Напряжения Рейнольдса предъявлены с необходимой для формулировки леммы точностью $\left(\bmod O\left(t^{3}\right)\right)$. 
$\Phi_{i}, i=0, \ldots, n$, определяются из уравнений $(17)$, а число $n$ может быть сколь угодно велико. В дальнейшем мы положим $n>2 N+8$.

Пусть функции $\mathscr{V}_{i}, \mathscr{R}_{i}, \mathscr{H}_{i}, \mathscr{T}_{i}, i=0, \ldots, n$, бесконечно дифференцируемы по всем своим аргументам и $2 \pi$-периодичны по $\eta$.

На данном этапе доказательства леммы мы предъявляем системы уравнений, которым должны удовлетворять коэффициенты разложения (13), чтобы исходная система при подстановке в нее приближенного решения $v_{n}, \rho_{n}, H_{n}, T_{n}, P_{n}$ выполнялась с точностью $O\left(\varepsilon^{n}\right)$ (см. $\left.[1,11,12]\right)$. Под выполнением уравнений с точностью $O\left(\varepsilon^{n}\right)$ подразумевается следующее: невязка имеет вид $\varepsilon^{n} F(\eta, x, t, \varepsilon, n)$, где функция $F$ удовлетворяет следующей оценке:

$$
|F| \leqslant \sum_{i=0}^{l} F_{i}(\eta, x, t, n) \varepsilon^{i} ;
$$

здесь $l$ - некоторое число, зависящее от $N$ и $\delta$, а $F_{i}, i=0, \ldots, l$, являются гладкими функциями, $2 \pi$-периодическими по $\eta$ и ограниченными по $t$ и $x$.

На втором этапе доказательства леммы при малом $t \in\left(0, t_{0}\right)$ предъявляется приближенное решение исходной задачи в виде конечного степенного ряда по $t$ :

$$
\begin{aligned}
& v_{n}(\eta, x, t, \varepsilon)= \sum_{i=0}^{r} v_{(i)}(\eta, x, \varepsilon) t^{i}, \quad \rho_{n}(\eta, x, t, \varepsilon)=\sum_{i=0}^{r} \rho_{(i)}(\eta, x, \varepsilon) t^{i}, \\
& H_{n}(\eta, x, t, \varepsilon)=\sum_{i=0}^{r} H_{(i)}(\eta, x, \varepsilon) t^{i}, \\
& T_{n}(\eta, x, t, \varepsilon)=\sum_{i=0}^{r} T_{(i)}(\eta, x, \varepsilon) t^{i}, \quad P_{n}(\eta, x, t, \varepsilon)=\sum_{i=0}^{r} P_{(i)}(\eta, x, \varepsilon) t^{i},
\end{aligned}
$$

где $r$ - некоторое число, зависящее от $N$ и $\delta$. Коэффициенты $v_{(i)}, \rho_{(i)}, H_{(i)}$, $T_{(i)}, P_{(i)}$ сами в свою очередь являются многочленами по $\varepsilon$.

Исходные уравнения при подстановке в них этого решения выполняются с точностью $O\left(\varepsilon^{n}\right)$, причем функции $F_{i}$ из (14) являются многочленами по $t$. При этом решение (15) при $t=0$ точно совпадает с начальными данными (7), (9)-(11).

На третьем этапе доказательства леммы мы корректируем полученное приближенное решение таким образом, чтобы уравнения (1)-(6) выполнялись точно.

Доказательство будет осуществлено для случая $\operatorname{Re}=\operatorname{Re}_{M}$ и $\varepsilon=1 / \sqrt{\operatorname{Re}}$, т.е. для $\beta=0$. Доказательство теоремы в случае $\operatorname{Re}_{M} \gg \operatorname{Re}$ и $\beta>0$ проводится аналогично.

Магнитное давление $P_{m}^{(n)}=P_{n}+\left|H_{n}\right|^{2} / 8 \pi$ также представляется в виде степенных рядов по $\varepsilon$ и $t$ :

$$
P_{m}^{(n)}=\mathscr{P}_{m}^{(0)}(\eta, x, t)+\cdots+\varepsilon \mathscr{P}_{m}^{(1)}(\eta, x, t)+\cdots+\varepsilon^{n+1} \mathscr{P}_{m}^{(n+1)}(\eta, x, t) .
$$

Зная разложения (13), (15) для плотности, температуры и вектора магнитной напряженности, без труда находим аналогичные разложения для гидродинамического и магнитного давлений $P_{n}$ и $P_{m}^{(n)}$. 
Пусть функции $\Phi_{0}(x, t), \Phi_{1}(x, t), \ldots, \Phi_{n}(x, t)$ бесконечно дифференцируемы и являются решениями следуюших задач Коши:

$$
\frac{\partial \Phi_{j}}{\partial t}+\sum_{i=0}^{j}\left\langle\left(v_{i}\right)_{\mathrm{av}}, \nabla \Phi_{j-i}\right\rangle=0,\left.\quad \Phi_{j}\right|_{t=0}=\left.\frac{1}{j !} \frac{\partial^{j} \Phi^{0}(x, \varepsilon)}{\partial \varepsilon^{j}}\right|_{\varepsilon=0}, \quad j=0, \ldots, n .
$$

Подставим решение (13) в исходную систему (1)-(6) и приравняем левые и правые части равенств при одинаковых степенях $\varepsilon$. При этом получаем замкнутые системы уравнений для нахождения сначала функций $\mathscr{V}_{0}, \mathscr{H}_{0}, \Phi_{0}, \mathscr{R}_{0}$, $\mathscr{P}_{0}, \mathscr{P}_{m}^{(0)}$ и $\mathscr{T}_{0}$, затем функций $\mathscr{V}_{1}, \mathscr{H}_{1}, \Phi_{1}, \mathscr{R}_{1}, \mathscr{P}_{1}$ и $\mathscr{T}_{1}$ и т.д. вплоть до нахождения функций $\mathscr{V}_{n}, \mathscr{H}_{n}, \Phi_{n}, \mathscr{R}_{n}, \mathscr{P}_{n}$ и $\mathscr{T}_{n}$.

В этих системах $\eta$ считается независимой переменной.

Если функции $\mathscr{V}_{i}, \mathscr{H}_{i}, \Phi_{i}, \mathscr{R}_{i}, \mathscr{P}_{i}, \mathscr{T}_{i}, i=0,1, \ldots, n$, являются решениями этих $(n+1)$ системы уравнений, то исходная система уравнений $(1)-(6)$ выполняется с точностью $O\left(\varepsilon^{n}\right)$.

Представим векторы $\mathscr{V}_{i}$ и $\mathscr{H}_{i}$ в виде суммы осредненных и осциллируюших частей: $\mathscr{V}_{i}=u_{i}+a_{i}, \mathscr{H}_{i}=B_{i}+h_{i}, i=0, \ldots, n$, где $u_{i}=\left(\mathscr{V}_{i}\right)_{\mathrm{av}}, B_{i}=\left(\mathscr{H}_{i}\right)_{\mathrm{av}}$.

Введем функции $\Lambda_{i}(\eta, x, t), c_{i}(x, t)$ следующим образом:

$$
\Lambda_{i}=\sum_{j=0}^{i+1}\left\langle a_{j}, \nabla \Phi_{i+1-j}\right\rangle, \quad c_{i}=\sum_{j=0}^{i+1}\left\langle B_{j}, \nabla \Phi_{i+1-j}\right\rangle, \quad i=0,1, \ldots, n .
$$

Коэффициенты $\mu, \zeta, \nu$ и $\varkappa$ мы также будем представлять в виде степенного ряда по $\varepsilon$, а в дальнейшем еще и в виде степенного ряда по $t: \mu=\mu_{0}+\varepsilon \mu_{1}+\ldots$, где $\mu_{0}=\mu\left(\mathscr{R}_{0}, \mathscr{T}_{0}\right), \mu_{1}=(\partial \mu / \partial \rho)\left(\mathscr{R}_{0}, \mathscr{T}_{0}\right) \mathscr{R}_{1}+(\partial \mu / \partial T)\left(\mathscr{R}_{0}, \mathscr{T}_{0}\right) \mathscr{T}_{1}, \ldots$, a $\zeta$, $\nu$ и $\varkappa$ разлагаются в ряд по $\varepsilon$ аналогично.

В работах $[11,12]$ выписаны системы уравнений для определения главных членов разложения $(13),(16)$. Они получаются подстановкой $(13),(16)$ в исходную систему (1)-(5) и приравниванием левой и правой частей полученных равенств при $\varepsilon^{-1}$ и $\varepsilon^{0}$.

В результате проведения такой процедуры получаем

$$
\begin{gathered}
\mathscr{R}_{0}\left(\frac{\partial \mathscr{V}_{0}}{\partial t}+\left\langle\mathscr{V}_{0}, \nabla\right\rangle \mathscr{V}_{0}\right)+\mathscr{R}_{0} \Lambda_{0} \frac{\partial \mathscr{V}_{0}}{\partial \eta} \\
=\frac{1}{4 \pi}\left(c_{0}-\int_{0}^{\eta} \operatorname{div} \mathscr{H}_{0} d \eta\right) \frac{\partial \mathscr{H}_{0}}{\partial \eta}-\nabla \Phi_{0} \frac{\partial \mathscr{P}_{m}^{(1)}}{\partial \eta} \\
-\nabla \mathscr{P}_{m}^{(0)}+\frac{1}{4 \pi}\left\langle\mathscr{H}_{0}, \nabla\right\rangle \mathscr{H}_{0}+\left|\nabla \Phi_{0}\right|^{2} \frac{\partial}{\partial \eta} \mu_{0} \frac{\partial \mathscr{V}_{0}}{\partial \eta}, \\
\frac{\partial \mathscr{R}_{0}}{\partial t}=-\mathscr{R}_{0} \frac{\partial \Lambda_{0}}{\partial \eta}-\operatorname{div}\left(\mathscr{V}_{0} \mathscr{R}_{0}\right),
\end{gathered}
$$

$$
\begin{aligned}
\frac{\partial \mathscr{H}_{0}}{\partial t}=\frac{\partial}{\partial \eta}\left(\left(c_{0}-\int_{0}^{\eta} \operatorname{div} \mathscr{H}_{0} d \eta\right)\right. & \left.\mathscr{V}_{0}-\Lambda_{0} \mathscr{H}_{0}\right) \\
& +\operatorname{rot}\left(\mathscr{V}_{0} \times \mathscr{H}_{0}\right)+\left|\nabla \Phi_{0}\right| \frac{\partial}{\partial \eta} \nu_{0} \frac{\partial \mathscr{H}_{0}}{\partial \eta},
\end{aligned}
$$

$$
\operatorname{div} B_{0}=0,
$$




$$
\begin{gathered}
\mathscr{R}_{0}\left(\frac{\partial \mathscr{T}_{0}}{\partial t}+\left\langle\mathscr{V}_{0}, \nabla\right\rangle \mathscr{T}_{0}+(\gamma-1) \mathscr{T}_{0}\left\langle\nabla, \mathscr{V}_{0}\right\rangle+(\gamma-1) \mathscr{T}_{0} \frac{\partial \Lambda_{0}}{\partial \eta}+\frac{\partial \mathscr{T}_{0}}{\partial \eta} \Lambda_{0}\right) \\
=\gamma\left|\nabla \Phi_{0}\right|^{2} \frac{\partial}{\partial \eta}\left(\varkappa_{0} \frac{\partial \mathscr{T}_{0}}{\partial \eta}+\frac{\nu_{0}}{4 \pi}\left\langle\frac{\partial \mathscr{H}_{0}}{\partial \eta}, \frac{\partial \mathscr{H}_{0}}{\partial \eta}\right\rangle+\mu_{0}\left\langle\frac{\partial \mathscr{V}_{0}}{\partial \eta}, \frac{\partial \mathscr{V}_{0}}{\partial \eta}\right\rangle\right), \\
\quad \frac{\partial \Phi_{0}}{\partial t}+\left\langle u_{0}, \nabla \Phi_{0}\right\rangle=0, \\
\frac{\partial c_{0}}{\partial t}+\operatorname{div} c_{0} u_{0}=\operatorname{div}\left(\int_{0}^{\eta} \operatorname{div} \mathscr{H}_{0} d \eta a_{0}+\Lambda_{0} \mathscr{H}_{0}+\nabla \Phi_{0}^{2} \mathscr{H}_{0} \frac{\partial \nu_{0}}{\partial \eta}\right) \\
\frac{\partial \mathscr{P}_{m}^{(0)}}{\partial \eta}=0, \quad\left\langle\nabla \Phi_{0}, a_{0}\right\rangle=0, \quad \mathscr{P}_{0}=k \mathscr{R}_{0} \mathscr{T}_{0}, \\
\mathscr{P}_{m}^{(0)}=\mathscr{P}_{0}+\frac{1}{8 \pi}\left\langle\mathscr{H}_{0}, \mathscr{H}_{0}\right\rangle, \\
\left.\mathscr{V}_{0}\right|_{t=0}=v^{0}(\eta, x, 0),\left.\quad \mathscr{H}_{0}\right|_{t=0}=B^{0}(x),\left.\quad \mathscr{R}_{0}\right|_{t=0}=\rho^{0}(\eta, x, 0), \\
\left.\mathscr{T}_{0}\right|_{t=0}=T^{0}(\eta, x, 0),\left.\quad \Phi_{0}\right|_{t=0}=\Phi^{0}(x, 0), \\
\left.c_{0}\right|_{t=0}=\left\langle\mathscr{H}_{1}^{0}(\eta, x, 0), \nabla \Phi^{0}(x, 0)\right\rangle_{\mathrm{av}} \cdot
\end{gathered}
$$

Приравнивая левые и правые части равенств, полученных подстановкой решения (13) в (1)-(5) при $\varepsilon^{j}, j=1, \ldots, n$, получаем замкнутые системы уравнений для нахождения $\mathscr{V}_{j}, \mathscr{R}_{j}, \mathscr{H}_{j}, \mathscr{T}_{j}, \mathscr{P}_{m}^{(j)}, j=1, \ldots, n$. Начальные данные для полученных уравнений полностью определяются начальными данными (7), (11) $[1,11]$.

Построив приближенные решения для каждой из таких систем уравнений для определения $\mathscr{V}_{j}, \mathscr{R}_{j}, \mathscr{H}_{j}, \mathscr{T}_{j}, \mathscr{P}_{j}, j=0, \ldots, n$, с точностью $O\left(\varepsilon^{n-j}\right)$, мы тем самым построим приближенное решение исходной системы (1)-(5) с точностью $O\left(\varepsilon^{n}\right)$.

Сначала построим приближенное решение задачи (18)-(26) в виде степенного ряда по $t$ вплоть до любой степени $t$. А именно, неизвестные функции будем искать в виде

$$
\begin{gathered}
\mathscr{V}_{0}=\sum_{i=0}^{n} \mathscr{V}_{0}^{i}(\eta, x) t^{i}, \quad \mathscr{R}_{0}=\sum_{i=0}^{n} \mathscr{R}_{0}^{i}(\eta, x) t^{i}, \\
\mathscr{H}_{0}=\sum_{i=0}^{n} \mathscr{H}_{0}^{i}(\eta, x) t^{i}, \quad \Phi_{0}=\sum_{i=0}^{n} \Phi_{0}^{i}(\eta, x) t^{i}, \quad \mathscr{T}_{0}=\sum_{i=0}^{n} \mathscr{T}_{0}^{i}(\eta, x) t^{i}, \\
\mathscr{P}_{0}=\sum_{i=0}^{n} \mathscr{P}_{0}^{i}(\eta, x) t^{i}, \quad c_{0}=\sum_{i=0}^{n} c_{0}^{i}(x) t^{i}, \quad \Lambda_{0}=\sum_{i=0}^{n} \Lambda_{0}^{i}(\eta, x) t^{i} .
\end{gathered}
$$

Положим

$$
\begin{gathered}
\mathscr{V}_{0}^{0}(\eta, x)=v^{0}(\eta, x, 0), \quad \mathscr{H}_{0}^{0}(\eta, x)=B^{0}(x), \quad \mathscr{R}_{0}^{0}(\eta, x)=\rho^{0}(\eta, x, 0), \\
\mathscr{T}_{0}^{0}(\eta, x)=T^{0}(\eta, x, 0), \quad \Phi_{0}^{0}(x)=\Phi^{0}(x, 0), \quad \mathscr{P}_{0}^{0}(\eta, x)=k \mathscr{R}^{0}(\eta, x) \mathscr{T}^{0}(\eta, x), \\
c_{0}^{0}(x)=\left(H_{1 \|}^{0}\right)_{\mathrm{av}}, \quad \Lambda_{0}^{0}(x)=\left.\Lambda_{0}\right|_{t=0} .
\end{gathered}
$$


Для проведения дальнейших выкладок нам удобнее переписать уравнения (18)-(26) в несколько ином виде, в частности выразив $\partial \mathscr{P}_{m}^{(1)} / \partial \eta$ через другие неизвестные функции этой системы [11]. Будем говорить, что скалярная или векторная функция принадлежит классу $\mathscr{K}$, если ее компоненты являются многочленами от функций $\mathscr{R}_{0}, \mathscr{T}_{0}, c_{0}, \mu_{0}, \zeta_{0}, \nu_{0}, \varkappa_{0}$, компонент $\mathscr{V}_{0}, \mathscr{H}_{0}$ и их производных и интегралов по $\eta$ и $x$. Заметим, что если функция $f(\eta, x, t)$ принадлежит $\mathscr{K}$, то она является бесконечно дифференцируемой по всем аргументам и $2 \pi$-периодической по $\eta$.

Выразим функцию $\Lambda_{0}$ через другие неизвестные функции системы (18)-(25). Для этого, используя зависимость давления от плотности и температуры, из уравнения непрерывности (2) получаем уравнение

$$
T \frac{\partial P}{\partial t}-P \frac{\partial T}{\partial t}+P T \operatorname{div} \mathscr{V}+T\langle\mathscr{V}, \nabla P\rangle-P\langle\mathscr{V}, \nabla T\rangle=0 .
$$

Подставляя в $(28)$ ряды (13) и группируя члены при $\varepsilon^{0}$, получим уравнение

$$
\frac{\partial \mathscr{P}_{0}}{\partial t}=-\frac{\partial \mathscr{P}_{0}}{\partial \eta} \Lambda_{0}-\mathscr{P}_{0} \frac{\partial \Lambda_{0}}{\partial \eta}+\frac{\mathscr{P}_{0}}{\mathscr{T}_{0}}\left(\frac{\partial \mathscr{T}_{0}}{\partial t}+\frac{\partial \mathscr{T}_{0}}{\partial \eta} \Lambda_{0}\right)+F_{1}, \quad \text { где } F_{1} \in \mathscr{K} .
$$

Учитывая уравнение (21), последнее уравнение можно переписать в виде

$$
\frac{\partial \mathscr{P}_{0}}{\partial t}=-\gamma \mathscr{P}_{0} \frac{\partial \Lambda_{0}}{\partial \eta}-\frac{\partial \mathscr{P}_{0}}{\partial \eta} \Lambda_{0}+F_{2}, \quad \text { где } F_{2} \in \mathscr{K} .
$$

Из уравнения (25) заключаем, что

$$
\mathscr{P}_{0}=-\frac{\left\langle\mathscr{H}_{0}, \mathscr{H}_{0}\right\rangle}{8 \pi}+C(x, t),
$$

где $C(x, 0)$ известно из начальных данных, а $\partial C(x, t) / \partial t$ будет найдено ниже.

Из последнего равенства получаем

$$
\frac{\partial \mathscr{P}_{0}}{\partial t}=-\frac{1}{4 \pi}\left\langle\frac{\partial \mathscr{H}_{0}}{\partial t}, \mathscr{H}_{0}\right\rangle+\frac{\partial C(x, t)}{\partial t} .
$$

Теперь мы можем выписать уравнение для нахождения функции $\Lambda_{0}$, используя уравнения $(30),(32)$ и $(20)$ :

$$
\begin{aligned}
\left(\gamma \mathscr{P}_{0}+\right. & \left.\frac{1}{4 \pi}\left\langle\mathscr{H}_{0}, \mathscr{H}_{0}\right\rangle\right) \frac{\partial \Lambda_{0}}{\partial \eta} \\
& +\left(\frac{\partial \mathscr{P}_{0}}{\partial \eta}+\frac{1}{4 \pi}\left\langle\frac{\partial \mathscr{H}_{0}}{\partial \eta}, \mathscr{H}_{0}\right\rangle\right) \Lambda_{0}+F_{3}+\frac{\partial C(x, t)}{\partial t}=0, \quad F_{3} \in \mathscr{K} .
\end{aligned}
$$

Заметим, что в силу формулы (31) коэффициент при $\Lambda_{0}$ в последнем уравнении равен нулю.

Теперь мы можем выразить $\Lambda_{0}$ через другие неизвестные функции системы (18)-(25):

$$
\Lambda_{0}=-\int_{0}^{\eta} \frac{F_{3}}{f} d \eta-\int_{0}^{\eta} \frac{\partial C / \partial t}{f} d \eta+C_{1}(x, t), \quad \text { где } f=\gamma \mathscr{P}_{0}+\frac{1}{4 \pi}\left\langle\mathscr{H}_{0}, \mathscr{H}_{0}\right\rangle .
$$


Заметим, что $f \geqslant$ const $>0$. Осталось найти $C_{1}$ и $\partial C / \partial t$. Поскольку $\left(\partial \Lambda_{0} / \partial \eta\right)_{\text {av }}$ $=0$, из уравнения $(33)$ получаем

$$
\frac{\partial C}{\partial t}=-\left(\frac{F_{3}}{f}\right)_{\mathrm{av}}\left(\frac{1}{f}\right)_{\mathrm{av}}^{-1} .
$$

Аналогичным образом из $(33),(34)$ и того, что $\left(\Lambda_{0}\right)_{\mathrm{av}}=0$, вытекает равенство

$$
C_{1}=\left(\int_{0}^{\eta} \frac{F_{3}}{f} d \eta+\left(\frac{F_{3}}{f}\right)_{\mathrm{av}}\left(\frac{1}{f}\right)_{\mathrm{av}}^{-1} \int_{0}^{\eta} \frac{d \eta}{f}\right)_{\mathrm{av}} .
$$

Его правая часть равна нулю, откуда, подставляя в (33) значение функции $F_{3}$, получаем

$$
\Lambda_{0}=\int_{0}^{\eta} \frac{F}{f} d \eta-\left(\frac{F}{f}\right)_{\mathrm{av}}\left(\frac{1}{f}\right)_{\mathrm{av}}^{-1} \int_{0}^{\eta} \frac{1}{f},
$$

где

$$
\begin{aligned}
F=\frac{1}{4 \pi}( & -\operatorname{div} \mathscr{H}_{0}\left\langle\mathscr{V}_{0}, \mathscr{H}_{0}\right\rangle+\left(c_{0}-\int_{0}^{\eta} \operatorname{div} \mathscr{H}_{0} d \eta\right)\left\langle\frac{\partial a_{0}}{\partial \eta}, \mathscr{H}_{0}\right\rangle \\
& \left.+\left\langle\operatorname{rot}\left(\mathscr{V}_{0} \times \mathscr{H}_{0}\right), \mathscr{H}_{0}\right\rangle+\frac{\partial}{\partial \eta} \mathscr{V}_{\left(\mathscr{R}_{0}, \mathscr{T}_{0}\right)\left\langle\frac{\partial \mathscr{H}_{0}}{\partial \eta}, \mathscr{H}_{0}\right\rangle}+(k \gamma-1) \mathscr{V}\left(\mathscr{R}_{0}, \mathscr{T}_{0}\right)\left|\frac{\partial \mathscr{H}_{0}}{\partial \eta}\right|^{2}\right) \\
& +k \gamma\left(\mathscr{R}_{0} \mathscr{T}_{0} \operatorname{div} \mathscr{V}_{0}+\left|\nabla \Phi_{0}\right|^{2} \frac{\partial}{\partial \eta}\left(\varkappa\left(\mathscr{R}_{0}\right) \frac{\partial \mathscr{T}_{0}}{\partial \eta}\right)+\mu\left(\mathscr{R}_{0}, \mathscr{T}_{0}\right)\left|\frac{\partial a_{0}}{\partial \eta}\right|^{2}\right) \\
& -k\left\langle\mathscr{V}_{0}, \nabla \mathscr{R}_{0} \mathscr{T}_{0}\right\rangle .
\end{aligned}
$$

При $t=0$ формула (35) дает ограничения (10). Теперь, подставив найденную функцию $\Lambda_{0}$ в систему (18)-(25), получим эволюционную систему уравнений

$$
\begin{gathered}
\frac{\partial \mathscr{V}_{0}}{\partial t}=F_{4}, \quad \frac{\partial \mathscr{R}_{0}}{\partial t}=F_{5}, \quad \frac{\partial \mathscr{H}_{0}}{\partial t}=F_{6}, \\
\frac{\partial \Phi_{0}}{\partial t}=\left\langle u_{0}, \nabla \Phi_{0}\right\rangle, \quad \frac{\partial c_{0}}{\partial t}=F_{7}, \quad \frac{\partial \mathscr{T}_{0}}{\partial t}=F_{8},
\end{gathered}
$$

где $F_{i} \in \mathscr{K}, i=4, \ldots, 8$.

Подставим в систему уравнений (36) многочлены (27) и приравняем коэффициенты в левой и правой частях равенств при $t$ в степени 0 , затем при $t$, затем при $t^{2}$ и т.д. Из полученных уравнений последовательно находим сначала $\mathscr{V}_{0}^{1}$, $\mathscr{R}_{0}^{1}, \mathscr{H}_{0}^{1}, \Phi_{0}^{1}, c_{0}^{1}, \mathscr{T}_{0}^{1}$, откуда станет известно и $\mathscr{P}_{0}^{1}$, затем $\mathscr{V}_{0}^{2}, \mathscr{R}_{0}^{2}, \mathscr{H}_{0}^{2}$, $\Phi_{0}^{2}, c_{0}^{2}, \mathscr{T}_{0}^{2}, \mathscr{P}_{0}^{2}$ и т.д.

При проведении такой процедуры важным является то, что все коэффициенты в формулах (27) оказываются финитными по $x$ функциями, за исключением, естественно, функций $\mathscr{R}_{0}^{0}, \mathscr{T}_{0}^{0}$ и $\mathscr{P}_{0}^{0}$, которые представимы в виде суммы положительной константы и финитной по $x$ функции. 
Аналогичным образом ищем функции $\mathscr{V}_{1}, \mathscr{R}_{1}, \mathscr{H}_{1}, T_{1}$ и $c_{1}$ в виде многочленов по $t$ :

$$
\begin{aligned}
& \mathscr{V}_{1}=\sum_{i=0}^{r_{1}} \mathscr{V}_{1}^{i}(\eta, x) t^{i}, \quad \mathscr{R}_{1}=\sum_{i=0}^{r_{1}} \mathscr{R}_{1}^{i}(\eta, x) t^{i}, \quad \mathscr{H}_{1}=\sum_{i=0}^{r_{1}} \mathscr{H}_{1}^{i}(\eta, x) t^{i}, \\
& \mathscr{T}_{1}=\sum_{i=0}^{r_{1}} \mathscr{T}_{1}^{i}(\eta, x) t^{i}, \quad c_{1}=\sum_{i=0}^{r_{1}} c_{1}^{i}(\eta, x) t^{i}, \quad \mathscr{P}_{1}=\sum_{i=0}^{r_{1}} \mathscr{P}_{1}^{i}(\eta, x) t^{i},
\end{aligned}
$$

где $r_{1}$ - некоторое число, зависящее от $N$ и $\delta$. Главные члены разложений $(37)$ находим из начальных условий.

Через $\mathscr{K}_{1}$ обозначим класс функций, в который входят многочлены от $\mathscr{R}_{0}$, $\mathscr{R}_{1}, \mathscr{T}_{0}, \mathscr{T}_{1}, c_{0}, c_{1}, \mathscr{P}_{0}, \mathscr{P}_{1}, \Lambda_{0}$, компонент $\mathscr{V}_{0}, \mathscr{V}_{1}, \mathscr{H}_{0}, \mathscr{H}_{1}$, а также их производных и интегралов по $\eta$ и $x$ и, кроме того, суммы таких многочленов, деленные на $\mathscr{R}_{0}, \mathscr{T}_{0},\left|\nabla \Phi_{0}\right|$ или $\mathscr{P}_{0}$.

Система уравнений для определения функций (37) имеет вид

$$
\begin{gathered}
\left(\frac{\partial \mathscr{V}_{1}}{\partial t}\right)=\frac{\partial \mathscr{V}_{0}}{\partial \eta} \Lambda_{1}+F_{1}^{1}, \quad \frac{\partial \mathscr{R}_{1}}{\partial t}=-\mathscr{R}_{0} \frac{\partial \Lambda_{1}}{\partial \eta}+F_{2}^{1}, \\
\frac{\partial \mathscr{H}_{1}}{\partial t}=-\frac{\partial}{\partial \eta}\left(\mathscr{H}_{0} \Lambda_{1}\right)+F_{3}^{1}, \quad \frac{\partial \Phi_{1}}{\partial t}=F_{4}^{1}, \quad \frac{\partial c_{1}}{\partial t}=\left(-\operatorname{div}\left(\mathscr{H}_{0} \Lambda_{1}\right)\right)_{\mathrm{av}}+F_{5}^{1}, \\
\frac{\partial T_{1}}{\partial t}=(1-\gamma) T_{0} \frac{\partial \Lambda_{1}}{\partial \eta}-\frac{\partial T_{0}}{\partial \eta} \Lambda_{1}+F_{6}^{1}, \quad \frac{\partial}{\partial \eta} \mathscr{P}_{1}=-\frac{1}{4 \pi} \frac{\partial}{\partial \eta}\left\langle\mathscr{H}_{0}, \mathscr{H}_{1}\right\rangle+F,
\end{gathered}
$$

где $F_{i}^{1}, F, i=1, \ldots, 5$, принадлежат $\mathscr{K}_{1}$. Функция $F$ известна из предыдущего построения $(F \in \mathscr{K})$.

Проделывая выкладки, совершенно аналогичные проведенным для нахождения $\Lambda_{0}$, получаем, что

$$
\left(\frac{1}{4 \pi}\left\langle\mathscr{H}_{0}, \mathscr{H}_{0}\right\rangle+\gamma \mathscr{P}_{0}\right) \frac{\partial \Lambda_{1}}{\partial \eta}+\left(\frac{1}{4 \pi}\left\langle\mathscr{H}_{0}, \frac{\partial \mathscr{H}_{0}}{\partial \eta}\right\rangle+\frac{\partial \mathscr{P}_{0}}{\partial \eta}\right) \Lambda_{1}+\frac{\partial C_{1}}{\partial t}+F_{8}=0,
$$

где $F_{8} \in \mathscr{K}_{1}$. Таким образом, для $\Lambda_{1}$ выполняется уравнение, отличающееся от уравнения для $\Lambda_{0}$ лишь правой частью, так что нахождение приближенного решения системы (38), а также систем для нахождения поправок более высоких порядков в виде многочленов от $t$ совершенно аналогично нахождению приближенного решения системы (18)-(25).

Таким образом, построено асимптотическое решение $\mathscr{V}_{n}, \rho_{n}, H_{n}, T_{n}, P_{n}$ задачи (1)-(6), имеющее вид

$$
\begin{gathered}
\mathscr{V}_{n}=\sum_{j=0}^{n} \sum_{i=0}^{r_{j}} \mathscr{V}_{j}^{i}\left(\frac{\Phi_{n}(x, t, \varepsilon)}{\varepsilon}, x\right) \varepsilon^{j} t^{i}, \quad \rho_{n}=\sum_{j=0}^{n} \sum_{i=0}^{r_{j}} \mathscr{R}_{j}^{i}\left(\frac{\Phi_{n}(x, t, \varepsilon)}{\varepsilon}, x\right) \varepsilon^{j} t^{i}, \\
H_{n}=\sum_{j=0}^{n} \sum_{i=0}^{r_{j}} \mathscr{H}_{j}^{i}\left(\frac{\Phi_{n}(x, t, \varepsilon)}{\varepsilon}, x\right) \varepsilon^{j} t^{i}, \quad T_{n}=\sum_{j=0}^{n} \sum_{i=0}^{r_{j}} \mathscr{T}_{j}^{i}\left(\frac{\Phi_{n}(x, t, \varepsilon)}{\varepsilon}, x\right) \varepsilon^{j} t^{i}, \\
\mathscr{P}_{n}=\sum_{j=0}^{n} \sum_{i=0}^{r_{j}} \mathscr{P}_{j}^{i}\left(\frac{\Phi_{n}(x, t, \varepsilon)}{\varepsilon}, x\right) \varepsilon^{j} t^{i}, \quad \text { где } \Phi_{n}(x, t, \varepsilon)=\sum_{j=0}^{n} \sum_{i=0}^{r_{j}} \Phi_{j}^{i}(x) \varepsilon^{j} t^{i},
\end{gathered}
$$


и $r_{j} \geqslant(n-j) / 2 \delta, j=0,1, \ldots, n$.

Важно, что в начальный момент асимптотическое решение $\mathscr{V}_{n}(x, 0, \varepsilon)$, $\rho_{n}(x, 0, \varepsilon), H_{n}(x, 0, \varepsilon), \mathscr{T}_{n}(x, 0, \varepsilon)$, как видно из его построения, точно совпадает с начальными данными $(7),(9)-(11)$.

Из способа построения асимптотического решения также следует, что коэффициенты $\mathscr{V}_{0}^{0}(\eta, x), \mathscr{V}_{0}^{1}(\eta, x)$ и другие в разложении $(39)$ являются $2 \pi$-периодическими по $\eta$ и финитными по $x$. Исключением, естественно, являются плотность и температура, которые представимы в виде суммы положительной константы и $2 \pi$-периодической по $\eta$ финитной по $x$ функции. Обозначим через $\mathscr{M}$ класс таких функций $F$, что $F=f_{0}(\eta, x, t)+\varepsilon f_{1}(\eta, x, t)+\cdots+\varepsilon^{l} f_{l}(\eta, x, t)$, где $\eta=\Phi_{0}(x, t) / \varepsilon+\Phi_{1}(x, t)+\cdots+\varepsilon^{l-1} \Phi_{l}(x, t)$, число $l$ зависит от $n$ и $\delta$, а функции $f_{i}, i=0, \ldots, l, 2 \pi$-периодичны по $\eta$, бесконечно дифференцируемы по всем аргументам, являются многочленами по $t$ и финитны по $x$. Сами функции $f_{i}$ могут зависеть от $n$ и $\delta$. Важно, что $F$ ограничена по $\varepsilon$ при $\varepsilon \in\left(0, \varepsilon_{0}\right)$.

Введем следующие обозначения:

$$
\begin{gathered}
L_{v}(\rho, v, H, T)=\rho\left(\frac{\partial v^{i}}{\partial t}+\langle v, \nabla\rangle v^{i}\right)-\frac{\partial}{\partial x_{i}}(k \rho T)-\frac{1}{4 \pi}(H \times \operatorname{rot} H)^{i} \\
+\varepsilon^{2} \frac{\partial}{\partial x_{k}}\left(\mu(\rho, T)\left(\frac{\partial v^{i}}{\partial x_{k}}+\frac{\partial v^{k}}{\partial x_{i}}-\frac{2}{3} \delta_{i k} \operatorname{div} v\right)\right)+\varepsilon^{2} \frac{\partial}{\partial x_{i}}(\zeta(\rho, T) \operatorname{div} v), \\
L_{\rho}(\rho, v)=\frac{\partial \rho}{\partial t}+\operatorname{div}(\rho v), \\
L_{H}(\rho, v, H, T)=\frac{\partial H}{\partial t}-\operatorname{rot}(v \times H)+\varepsilon^{2} \operatorname{rot}(\nu(\rho, T) \operatorname{rot} H), \\
L_{T}(\rho, v, H, T)=\rho\left(\frac{\partial T}{\partial t}+\langle v, \nabla T\rangle\right)-\left((1-\gamma) \rho T \operatorname{div} v+\varepsilon^{2} \operatorname{div}(\varkappa(\rho) \nabla T)\right. \\
+\varepsilon^{2} \frac{\nu(\rho, T)}{4 \pi}(\operatorname{rot} H)^{2}+\varepsilon^{2} \frac{\mu(\rho, T)}{2} \frac{\partial v^{i}}{\partial x_{k}}\left(\frac{\partial v^{i}}{\partial x_{k}}+\frac{\partial v^{k}}{\partial x_{i}}-\frac{2}{3} \delta_{i k} \operatorname{div} v\right) \\
\left.+\varepsilon^{2} \zeta(\rho, T)(\operatorname{div} v)^{2}\right) .
\end{gathered}
$$

Подставив построенное асимптотическое решение в исходные уравнения, получаем

$$
\begin{gathered}
L_{v}=\left(\rho_{n}, v_{n}, H_{n}, T_{n}\right)=\varepsilon^{n} f_{1}, \quad L_{\rho}\left(\rho_{n}, v_{n}\right)=\varepsilon^{n} f_{2}, \\
L_{H}\left(\rho_{n}, v_{n}, H_{n}, T_{n}\right)=\varepsilon^{n} f_{3}, \quad \operatorname{div} H_{n}=\varepsilon^{n} f_{n}, \\
L_{T}\left(\rho_{n}, v_{n}, H_{n}, T_{n}\right)=\varepsilon^{n} f_{5}, \quad P_{n}=k \rho_{n} T_{n},
\end{gathered}
$$

где $f_{i} \in \mathscr{M}, i=1, \ldots, 5$.

Заметим, что начальные данные (7), (11) для построенного асимптотического решения выполнены точно. Рассмотрим новое асимптотическое решение $\tilde{\rho}_{n}, v_{n}$, $H_{n}, T_{n}$, где

$$
\tilde{\rho}_{n}\left(\frac{\Phi_{n}(x, t, \varepsilon)}{\varepsilon}, x, t, \varepsilon\right)=\rho_{n}\left(\frac{\Phi_{n}(x, t, \varepsilon)}{\varepsilon}, x, t, \varepsilon\right)+\varepsilon^{n} \tilde{\rho}\left(\frac{\Phi_{n}(x, t, \varepsilon)}{\varepsilon}, x, t, \varepsilon\right),
$$


а $\tilde{\rho}$ является решением уравнения, выписанного ниже. Пусть $\rho_{1}=\tilde{\rho}(\eta, x, t, \varepsilon)$, где $\eta$ считается независимой переменной.

Подставим новое решение в уравнение непрерывности:

$$
\begin{aligned}
L_{\rho}\left(\tilde{\rho}_{n}, v_{n}\right)=L_{\rho}\left(\rho_{n}, v_{n}\right)+\varepsilon^{n}\{ & \frac{\partial \rho_{1}}{\partial t}+\frac{1}{\varepsilon} \frac{\partial \rho_{1}}{\partial \eta} \frac{\partial \Phi_{n}}{\partial t}+\operatorname{div}\left(\rho_{1} v_{n}\right) \\
& \left.+\frac{1}{\varepsilon} \frac{\partial \rho_{1}}{\partial \eta}\left\langle v_{n}, \nabla \Phi_{n}\right\rangle+\frac{1}{\varepsilon} \rho_{1}\left\langle\frac{\partial v_{n}}{\partial \eta}, \nabla \Phi_{n}\right\rangle\right\} .
\end{aligned}
$$

Разлагая выражения $\partial \Phi_{n} / \partial t+\left\langle v_{n}, \nabla \Phi_{n}\right\rangle$ и $\left\langle\partial v_{n} / \partial \eta, \nabla \Phi_{n}\right\rangle$ в степенной ряд по $\varepsilon$ и используя формулы $(22),(23)$, получаем

$$
\frac{\partial \Phi_{n}}{\partial t}+\left\langle v_{n}, \nabla \Phi_{n}\right\rangle=\varepsilon \varphi_{1}(\eta, x, t, \varepsilon), \quad\left\langle v_{n}, \nabla \Phi_{n}\right\rangle=\varepsilon \varphi_{2}(\eta, x, t, \varepsilon),
$$

где $\varphi_{1}$ и $\varphi_{2}$ принадлежат $\mathscr{M}$.

Пусть $\rho_{1}$ - решение линейной задачи Коши

$$
\frac{\partial \rho_{1}}{\partial t}+\varphi_{1} \frac{\partial \rho_{1}}{\partial \eta}+\operatorname{div}\left(v_{n} \rho_{1}\right)+\varphi_{2} \rho_{1}=-f_{2},\left.\quad \rho_{1}\right|_{t=0}=0 .
$$

Заметим, что функции $\varphi_{1}, v_{n}, \varphi_{2}$ и $f_{2}$ являются многочленами по $\varepsilon$, и, следовательно, функцию $\rho_{1}$ мы тоже ищем в виде многочлена по $\varepsilon$, решая вместо одной задачи (42) конечный ряд задач, получающихся приравниванием коэффициентов при каждой степени $\varepsilon$ в левой и правой частях уравнения (42).

Подставив новое решение $\tilde{\rho}_{n}, v_{n}, H_{n}, T_{n}$ в исходную систему (1)-(6), получим

$$
\begin{gathered}
L_{v}\left(v_{n}, \tilde{\rho}_{n}, H_{n}, T_{n}\right)=L_{v}\left(v_{n}, \rho_{n}, H_{n}, T_{n}\right)+\varepsilon^{n-1} f_{1}^{1}=\varepsilon^{n-1} f_{1}^{2}, \\
L_{\rho}\left(v_{n}, \tilde{\rho}_{n}\right)=0, \quad L_{H}\left(v_{n}, \tilde{\rho}_{n}, H_{n}, T_{n}\right)=\varepsilon^{n-1} f_{3}^{1}, \\
\operatorname{div} H_{n}=\varepsilon^{n} f_{n}, \quad L_{T}\left(v_{n}, \tilde{\rho}_{n}, H_{n}, T_{n}\right)=\varepsilon^{n-1} f_{5}^{1},
\end{gathered}
$$

где $f_{1}^{1}, f_{1}^{2}, f_{3}^{1}, f_{5}^{1}$ принадлежат классу $\mathscr{M}$.

Следуюшим шагом еще подкорректируем приближенное решение, добавляя малую величину порядка $O\left(\varepsilon^{n}\right)$ уже к вектору магнитной напряженности: $\widetilde{H}_{n}=$ $H_{n}+\varepsilon^{n} h$, где $h=\left(-\int_{0}^{x} f_{4} d x, 0,0\right)$. Так как $\left.f_{4}\right|_{t=0}=0$, то и $\left.h\right|_{t=0}=0$.

Приближенное решение $v_{n}, \tilde{\rho}_{n}, \widetilde{H}_{n}, T_{n}$ удовлетворяет следующей системе уравнений:

$$
L_{v}\left(v_{n}, \tilde{\rho}_{n}, \tilde{H}_{n}, T_{n}\right)=\varepsilon^{n-2} f_{1}^{3}, \quad L_{\rho}\left(v_{n}, \tilde{\rho}_{n}\right)=0
$$

$L_{H}\left(v_{n}, \tilde{\rho}_{n}, \widetilde{H}_{n}, T_{n}\right)=\varepsilon^{n-2} f_{3}^{2}, \quad \operatorname{div} \widetilde{H}_{n}=0, \quad L_{T}\left(v_{n}, \tilde{\rho}_{n}, \widetilde{H}_{n}, T_{n}\right)=\varepsilon^{n-1} f_{5}^{2}$,

где $f_{1}^{3}, f_{3}^{2}, f_{5}^{2}$ принадлежат классу $\mathscr{M}$.

Еще раз подкорректируем решение, добавив к вектору магнитной напряженности вектор $B$, где $B\left(\Phi_{n}(x, t, \varepsilon) / \varepsilon, x, t\right)$ является решением следующей задачи Коши:

$$
L_{H}\left(v_{n}, \tilde{\rho}_{n}, B, T_{n}\right)=-\varepsilon^{n-2} f_{3}^{2},\left.\quad B\right|_{t=0}=0
$$


Из утверждений работы [3] следует, что решение $B$ задачи (43) удовлетворяет неравенству $\max _{0 \leqslant t \leqslant t_{0}}|B| \leqslant \varepsilon^{n-5} c(x)$, где $c(x)$ принадлежит $\mathscr{M}$ и может зависеть от $n$.

Взяв дивергенцию от обеих частей уравнения (43), в силу того что $\operatorname{div} f_{3}^{2}=0$, получим равенство $\operatorname{div} B=0$.

Обозначим $\widetilde{H}_{n}+B$ через $\widetilde{\widetilde{H}}_{n}$ и подставим новое решение $v_{n}, \tilde{\rho}_{n}, \widetilde{\widetilde{H}}_{n}, T_{n}$ в исходную систему. Таким образом, мы получаем $L_{v}=\varepsilon^{n-3} f_{1}^{4}, L_{\rho}=L_{H}=$ $\operatorname{div} \widetilde{\widetilde{H}}_{n}=0, L_{T}=\varepsilon^{n-6} f_{5}^{3}$, где $f_{1}^{4} \in \mathscr{M}, f_{5}^{3} \in \mathscr{M}$.

И наконец, добавим малую функцию $\varepsilon^{n-6} \widetilde{T}$ к температуре, добиваясь тем самым точного выполнения уравнения теплопроводности (5). Пусть $\left.\widetilde{T}\right|_{t=0}=0$ и $T_{1}=\widetilde{T}(\eta, x, t, \varepsilon)$ удовлетворяет уравнению

$$
\begin{aligned}
& \tilde{\rho}_{n}\left(\frac{\partial T_{1}}{\partial t}+\right.\left.\frac{\partial T_{1}}{\partial \eta} \varphi_{1}+\left\langle v_{n}, \nabla T_{1}\right\rangle+(\gamma-1) \varphi_{2} T_{1}\right) \\
&=\varepsilon \varkappa\left(\tilde{\rho}_{n}\right)\left\langle\nabla T_{1}, \nabla \Phi_{n}\right\rangle+\varepsilon^{2} \operatorname{div}\left(\varkappa\left(\tilde{\rho}_{n}\right) \nabla T_{1}\right) \\
&+\left(\frac{1}{4 \pi} \frac{\partial \nu}{\partial T}\left(\tilde{\rho}_{n}\right)(\operatorname{rot} \widetilde{\widetilde{H}})^{2}+\frac{1}{2} \frac{\partial \mu}{\partial t}\left(\tilde{\rho}_{n}\right) \frac{\partial v_{n}^{i}}{\partial x_{k}}\left(\frac{\partial v_{n}^{i}}{\partial x_{k}}+\frac{\partial v_{n}^{k}}{\partial x_{i}}-\frac{2}{3} \delta_{i k} \operatorname{div} v_{n}\right)\right. \\
&\left.+\frac{\partial \zeta}{\partial T}\left(\tilde{\rho}_{n}\right)(\operatorname{div} v)^{2}\right) T_{1}-f_{5}^{3},
\end{aligned}
$$

где $\varphi_{1}$ и $\varphi_{2}$ определены в (41). Отметим, что в случае произвольной гладкой зависимости коэффициентов $\mu, \zeta, \nu$ и $\varkappa$ от температуры потребуются более громоздкие оценки.

Окончательно для решения $v_{n}, \tilde{\rho}_{n}, \widetilde{\widetilde{H}}_{n}, \widetilde{T}_{n}$ получаем $L_{v}=\varepsilon^{n-8} f_{1}^{5}, L_{\rho}=$ $L_{H}=\operatorname{div} \widetilde{\widetilde{H}}_{n}=L_{T}=0, f_{1}^{5} \in \mathscr{M}$.

В формулировке теоремы зафиксировано сколь угодно большое $N$. В нашем построении приближенного решения число $n$ может быть сколь угодно велико. Положим $n>2 N+8$. Теперь ясно, что предъявленное решение является решением, фигурирующим в формулировке теоремы. Осредняя по быстрой переменной уравнения (18)-(23), получим уравнения Рейнольдса. Доказательство леммы завершено.

Выражаю глубокую благодарность И. Князевой за огромную помощь.

\section{ЛитеРАтУРА}

1. Маслов В. П., Омельянов Г. А. Однофазовая асимптотика для уравнений магнитной гидродинамики при больших числах Рейнольдса, Сиб. матем. ж., 29, №5, 172-180 (1988).

2. Maslov V. P., Omel' yanov G. A. The dynamo problem for large hydrodynamic and magnetic Reynolds numbers. Russian J. Math. Phys., 4, No. 1, 75-86 (1996).

3. Шафаревич А. И. Поведение магнитного поля в проводящей жидкости с быстро меняющимся полем скоростей. Докл. РАН, в печати.

4. Maslov V. P. Improper character of the ideal charged gas equations and vortex turbulence, in: Plasma Theory and Nonlinear and Turbulent Processes in Physics, Proceedings of the International Workshop (V. G. Bar'yakhtar et al., eds.), Vol. II, Kiev, pp. 692-717. 
5. Zel'dovich Ya. B., Ruzmaikin A. A., Molchanov S. A., Sokolov D. D. Kinematic dynamo problem in a linear velocity field. J. Fluid Mech., 144, 1-11 (1984).

6. Sovard A. M., Childress S. Dynamo action at large magnetic Reynolds numbers in spatially periodic flow with mean motion, in: Topological Fluid Mech., Proc. of IUTAM Symposium (H. K. Moffat and A. Tsinober, eds.), Cambridge (1989).

7. Gailitis $A$. The Helical MHD Dynamo, in: Topological Fluid Mech., Proc. of IUTAM Symposium (H. K. Moffat and A. Tsinober, eds.), Cambridge (1989).

8. Rädler K.-H., Seehafer N. Relations between helicities in mean-field dynamo models, in: Topological Fluid Mech., Proc. of IUTAM Symposium (H. K. Moffat and A. Tsinober, eds.), Cambridge (1989).

9. Ho Y. L., Prager S. C. Boundary effects on the MHD dynamo in laboratory plasmas, in: Topological Fluid Mech., Proc. of IUTAM Symposium (H. K. Moffat and A. Tsinober, eds.), Cambridge (1989).

10. Доброхотов С. Ю., Оливе В. Мартинес. Локализованные асимптотические решения уравнения магнитного динамо в АВС-полях. Матем. заметки, 54, №4, 45-68 (1993).

11. Maslov V. P., Omel'yanov G. A. Fluctuation-generated tokamak pinch instabilities. Russian J. Math. Phys., 2, No. 4, 463-485 (1994).

12. Маслов В. П., Омельянов Г. А. Нелинейная эволюция флуктуаций. Физика плазмы, 21, №8, 684-696 (1995).

Московский государственный

Поступило в редакцию

институт электроники и математики

15 июня 1996 г. 